\title{
Los Sistemas de Referencia y Contrarreferencia de pacientes en América Latina: Mecanismos de Coordinación Asistencial y el rol de la Medicina Familiar y Comunitaria
}

\author{
Referral and Counter-Referral Patient Management Systems in Latin America: Care Coordination \\ Mechanisms and the role of Family and Community Medicine

\section{Os Sistemas de Referencia e Contra-Referência de pacientes na América Latina: Mecanismos de Coordenação Assistencial e papel da Medicina de Família e Comunidade}

\author{
Miguel Ángel Fernández Ortega. Facultad de Medicina, Universidad Nacional Autónoma de México (UNAM); Subregión Mesoamérica de \\ Confederación Iberoamericana de Medicina Familiar (WONCA Iberoamericana-CIMF). Ciudad de México, México. miguelaf03@live.com \\ (Autor correspondiente) \\ Jacqueline Beatriz Roo Prato. Facultad de Medicina, Universidad Nacional Autónoma de México (UNAM). Ciudad de México, México. \\ jroop3@gmail.com \\ Arnulfo Emanuel Irigoyen Coria. Facultad de Medicina, Universidad Nacional Autónoma de México (UNAM); Academia Nacional de \\ Medicina de México. Ciudad de México, México.coriaa@unam.mx \\ Sofía Blanco Lara. Asociación de Medicina Familiar de Costa Rica. Costa Rica. sifiablanco@yahoo.com \\ Alan Edward Barrell. Ex Asesor de la Facultad de Medicina, Universidad Autónoma de Guadalajara. México. aebarrell@yahoo.com \\ Víctor Manuel Juárez Téllez. Facultad de Medicina, Universidad Nacional Autónoma de México (UNAM); Instituto Mexicano del Seguro \\ Social (IMSS); Representante por Waynakay. México. victorit82@gmail.com
}

\section{Grupo de Trabajo}

Alexis Correa Taja (Nicaragua); Ana Margarita Muñiz Roque (Cuba); Ana Lucía Meyer (República Dominicana); Ariel Cruz lgartua (Puerto Rico); Cecilia Llorach (Panamá); Guillermo Franco Gil (México); Grace Bethel Aparicio (El Salvador); Mariela Rodríguez (Chile); Miguel Ángel Fernández Ortega (México); Sofía Blanco Lara (Costa Rica); María Alicia Macedo Cantero (Paraguay); Neimaru Abreu (Venezuela); Victor Salvador Narcizo Zarate (Perú); Paulo Poli (Brasil); Miguel Ángel Suárez Cuba (Bolivia); María del Rosario Caballé (Argentina); Víctor Manuel Juárez Téllez (México); Jorge López Gálvez (Chile); Daniel Ulate Gómez (Costa Rica); Karla Patricia Jiménez Ríos (Costa Rica); Ma. Alicia Macedo (Paraguay); Pablo Armijos Peña (Ecuador); Ysabel María Díaz Brito (República Dominicana); Flavia Solórzano Morera (Costa Rica); Melba Váques Escalante (Costa Rica); Adriana Villafranca Brenes (Costa Rica); Willy Loria Quiros (Costa Rica); Roberto Cerda (República Dominicana); José Adalberto Mejía (Honduras); Ada Alicia López (Honduras); Aron Hernández Cervantes (Costa Rica); Joao Werner Falk (Brasil); Ricardo Fabrega (Chile)

Cómo citar: Fernández MA, Roo JP, Irigoyen AC, Blanco SL, Edward AB, Juárez VT. Los Sistemas de Referencia y Contrarreferencia de pacientes en América Latina: Mecanismos de Coordinación Asistencial y el rol de la Medicina Familiar y Comunitaria. Rev Bras Med Fam Comunidade. 2016;11(Suppl 2):37-45. http://dx.doi.org/10.5712/rbmfc11(0)1384
Financiación:

ninguna declarada.

Aprobación ética:

La investigación fue

considerada una investigación

sin riesgo. Los autores declaran

que los procedimientos

seguidos se realizaron de

acuerdo a las normas éticas de

la Asociación Médica Mundial y

la Declaración de HelsinKi.

Conflicto de intereses:

ninguna declarada.

Procedencia y revisión por pares:

revisado por pares.

Recibido el: 15/08/2016.

Aceptado el: 15/09/2016. 


\section{Resumen}

Se presentan los resultados del diagnóstico situacional de 16 países de América Latina, en cuanto a sus Sistemas de Referencia y Contrarreferencia de pacientes entre los tres niveles de atención médica. Los resultados de la investigación fueron analizados por representantes de los países miembros de WONCA Iberoamérica CIMF, en el marco de la VI Cumbre Iberoamericana de Medicina Familiar en San José de Costa Rica en abril de 2016.

\section{Abstract}

The results of the Situational Analysis among their various levels of healthcare of 16 countries in Latin America related to their Referral and Counter-Referral Patient Management Systems are presented in this document. The research results were analyzed by WONCA Latin America country member representatives at the VI Ibero-American Family Medicine Summit in San Jose, Costa Rica in April 2016.

\section{Resumo}

São apresentados os resultados do diagnostico situacional de 16 países da América Latina em relação aos seus Sistemas de Referência e Contra-Referencia de pacientes entre os três níveis de atenção médica. Os resultados da pesquisa foram analisados por representantes dos países membros da WONCA Ibero-Americana CIMF no âmbito da VI Cúpula Ibero-Americana de Medicina Familiar em San Jose, Costa Rica em abril 2016.

\section{Palabras clave:}

Integración

Primaria/Secundaria

Sistemas de Salud

Atención Primaria de salud

Medicina Familiar

Keywords:

Primary/Secondary Integration

Health Systems

Primary Healthcare

Family Medicine

Palavras-chave:

Integração Primária/Secundária

Sistemas de Saúde

Atenção Primária de saúde

Medicina de Família

\section{Introducción}

Según la Organización Mundial de la Salud (OMS), los gobiernos de todo el mundo tienen la responsabilidad de garantizar que sus sistemas de salud respondan a las necesidades de su población con eficacia y eficiencia, basando sus modelos de salud en la Atención Primaria (APS). En función de esta premisa, los Estados Miembros se comprometieron en $\mathbf{2 0 0 5}$ a desarrollar sus sistemas de financiación sanitaria, de manera que todas las personas tuvieran acceso a los servicios y no sufrieran dificultades financieras al pagar por ellos, a este objetivo se le definió como "Cobertura Universal". ${ }^{1}$

Según Margaret Chan, directora de OMS, "entre el 20\% y el $40 \%$ del gasto en salud se pierde por la ineficiencia en los sistemas de salud". La falta de planeación en la prestación de servicios, la duplicidad de recursos, el ineficiente sistema de registro e información, la falta de recursos humanos debidamente formados y capacitados llevan a la ineficacia de las redes de prestación de servicios, generando graves problemas de inequidad, acceso y cobertura que conducen a la muerte a muchas personas cada año. ${ }^{1}$

La OMS plantea que un sistema de salud "consiste en el conjunto de organizaciones, personas y acciones cuya finalidad es promover, restaurar o mantener la salud". ${ }^{2-4}$

En 2011, al respecto de la 64a Asamblea Mundial de Salud de la OMS, en lo referente a los Modelos de prestación integrada de servicios, el acuerdo final fue redactado de la siguiente manera:

"La organización de la atención sanitaria está adoptando la forma de "redes de prestación integrada de servicios". Adaptando la experiencia adquirida en el desarrollo de sistemas de salud de distrito a los sistemas de salud pluralistas, las redes de prestación integrada de servicios se organizan como redes de proveedores de atención primaria próximas al cliente - públicas, privadas o mixtas, respaldadas por hospitales y servicios especializados. Estas redes se responsabilizan de la salud de una población definida, ofreciendo servicios de promoción de la salud, prevención de enfermedades, diagnóstico, tratamiento, manejo de la morbilidad, rehabilitación y asistencia paliativa...".1,5

En este sentido, la OMS, menciona en el documento "La Renovación de la Atención Primaria de Salud en las Américas", de 2007: 
"Los servicios de atención primaria deben ser apoyados y complementados por diferentes niveles de atención especializada, tanto ambulatorios como hospitalarios, así como por el resto de la red de protección social. Por tal razón, los sistemas de atención de salud deben trabajar de forma integrada mediante el desarrollo de mecanismos de coordinación de la atención a lo largo de todo el espectro de servicios, incluyendo el desarrollo de redes y de sistemas de referencia y contrarreferencia...". ${ }^{6}$

Los sistemas nacionales de salud en muchos de los países del mundo, se han organizado bajo la estrategia de niveles de atención médica o escalonamiento de los servicios dentro de los cuales encontramos las unidades operativas del primer nivel (clínicas, unidades de Medicina Familiar o Salud Familiar, centros de salud, etc.), que brindan atención específica y de carácter ambulatorio, resuelve del 80 a $85 \%$ de los problemas de salud más frecuentes, con tecnología de baja complejidad. ${ }^{7-16}$

Los hospitales generales, clínicas de especialidades o policlínicos que corresponden al segundo nivel, son responsables de resolver el 10-15\% de los problemas de salud referidos por el primer nivel de atención o consultados espontáneamente a través de los servicios de Urgencias y requieren tecnología de mediana complejidad. ${ }^{14-16}$

El tercer nivel de atención lo conforman "Los Institutos Nacionales de Salud y Centros Médicos de Especialidades los cuales están diseñados para resolver el $5 \%$ de los problemas de salud que requieren tecnología de alta complejidad..."14-16

La integración entre niveles de atención, funcionando como red coordinada de servicios de salud, favorecen la continuidad e integralidad en la prestación de servicios, que en la mayor parte de los países se traducen en Sistemas de Referencia y Contrarreferencia de Pacientes (SRCRP). ${ }^{17}$

Se define al SRCRP como el procedimiento de coordinación entre las unidades operativas de los tres niveles de atención médica para facilitar el envío y recepción de pacientes, con el propósito de brindar atención médica oportuna, integral y de calidad, que va más allá de los límites regionales y de los ámbitos institucionales para garantizar el acceso a los servicios de salud en beneficio del paciente referido. ${ }^{7,8,11-14}$

El SRCRP está organizado de acuerdo a las necesidades de cada país en dos formas básicamente: geográfica o institucional, aunque puede haber una mezcla de ambas. Podría decirse que existen diferentes niveles de estructura organizacional, que van desde el nivel nacional o federal; estatal y local (municipal o de alcaldía), variando la eficacia y eficiencia del Sistema, principalmente en la ejecución de los procesos, principalmente de contrarreferencia. ${ }^{7,8,11,12,17-20}$

\section{Objetivo}

Determinar la situación que guardan los Sistemas de Referencia y Contrarreferencia de pacientes en los sistemas de salud de los países de Iberoamérica, con el propósito de identificar estrategias que permitan fortalecer la coordinación asistencial vinculada a la práctica de la Medicina Familiar en los países de la Región.

\section{Material y Métodos}

Estudio exploratorio, transversal, con fines de diagnóstico situacional, se realizó entre los meses de octubre de 2015 a marzo de 2016. Se convocó a participar a los presidentes de las sociedades científicas de Medicina Familiar de los 20 países miembros de WONCA Iberoamericana-CIMF, a través de uno o dos representantes expertos en este tema. Dentro de sus participantes, se encontraron académicos, investigadores, clínicos y miembros de las juntas directivas de las sociedades científicas nacionales, los cuales se dieron a la tarea de responder un cuestionario con 33 preguntas relacionadas con el Sistema de Referencia y Contrarreferencia en sus respectivos países. Dicho cuestionario fue previamente revisado y validado en su contenido por profesores de diferentes instituciones educativas de México y Costa Rica, y en un segundo momento por el grupo de participantes en el estudio. El cuestionario fue enviado vía 
correo electrónico a cada uno de los participantes e integradas las respuestas por país. Al finalizar la integración del informe final fue enviado a todos los integrantes del grupo para su revisión y aprobación de la información, la cual se presentó en las mesas de trabajo durante la VI Cumbre Iberoamericana de Medicina Familiar en San José de Costa Rica en el mes de abril de 2016 para su análisis y discusión.

\section{Resultados}

Se obtuvo respuesta de 16 de los 20 países: Argentina, Bolivia, Brasil, Chile, Colombia, Costa Rica, Cuba, El Salvador, México, Nicaragua, Panamá, Paraguay, Perú, Puerto Rico, República Dominicana y Venezuela. No participaron: Ecuador, España, Portugal ni Uruguay.

A continuación se describen los resultados encontrados.

\section{Los modelos de salud que cuentan con especialistas en Medicina Familiar}

Los 16 países encuestados respondieron que consideran al médico familiar en sus modelos de atención, en algunos con mayor grado de consolidación como es el caso de Cuba, Brasil y México. En otros como Panamá, Perú, Paraguay, Costa Rica, República Dominicana y Colombia, se observan avances considerables en función de las nuevas reformas a los sistemas de salud, que consideran al modelo de Medicina Familiar y Salud Familiar como piedra angular de sus Modelos de Salud.

\section{Los niveles de atención en los países de América Latina}

De los 16 países encuestados, el cien por ciento reportó contar con tres niveles de atención clasificados de acuerdo al grado de complejidad, donde el primer nivel de atención corresponde a la actividad asistencial con menor infraestructura, de mayor cobertura y de tipo generalista. En el caso de Colombia el sistema de salud también describe tres niveles de atención, sin embargo, en el ambiente asistencial, se habla de "un cuarto nivel", que en realidad corresponde al tercer nivel de atención.

\section{Función del Médico Familiar en los niveles de atención}

Los especialistas en Medicina Familiar en los 16 países laboran principalmente en el nivel primario. Cuba, Brasil, Panamá y Venezuela reportan una participación clínica y administrativa casi exclusiva en el primer nivel de atención. Los otros 11 países como Argentina, Bolivia (Caja Nacional de Salud), Chile, Colombia, El Salvador, México, Nicaragua, Paraguay, Perú y República Dominicana, reportan un predominio de la asignación asistencial del médico familiar en el primer nivel de atención, no obstante, también se le puede ubicar en el segundo, dedicado a la atención de urgencias hospitalarias, atención de padecimientos crónico degenerativos, cuidados paliativos y funciones administrativas. Sólo en los casos de Costa Rica y Puerto Rico, se refirieron como principal ambiente laboral, las labores administrativas y de gestión médica (Tabla 1).

Tabla 1. Ubicación de los médicos familiares según sus funciones en los Niveles de Atención

\begin{tabular}{|ll|}
\hline 1er nivel (clínica y de gestión)* $^{*}$ & Cuba, Brasil, Panamá, Venezuela \\
$1^{\circ}$ y $2^{\circ}$ nivel (clínica, gestión y guardias hospitalarias en & Argentina, Bolivia, Chile, Colombia, El Salvador, México, \\
salas de urgencias)* & Nicaragua, Paraguay, Perú, República Dominicana. \\
$1^{\circ}, 2^{\circ}$ y $3 e r$ nivel (labores administrativas y de gestión medica)* & Costa Rica y Puerto Rico \\
Docencia & Todos (excepto Nicaragua) \\
Investigación* & Ninguno \\
\hline
\end{tabular}

*como actividad prioritaria. 


\section{Existencia de SRCRP}

Los 16 países encuestados cuentan con SRCRP, de ellos, 13 lo tienen bien definido a nivel nacional, encontrando cuatro denominaciones como las más frecuentes: Sistema de Referencia y Contrarreferencia de pacientes (Bolivia, Brasil, Colombia, Chile, Cuba, El Salvador, México, Paraguay, Perú, Puerto Rico, República Dominicana, Venezuela) y en Panamá se le conoce como Sistema Único de Referencia y Contrarreferencia (SURCO). Argentina, Colombia y Perú no cuentan con un sistema consolidado de carácter nacional, más bien funciona a nivel institucional. En el caso de Costa Rica, Argentina y El Salvador no cuentan con un marco jurídico preciso, sin embargo, los Ministerios de Salud trabajan en la conformación de redes integradas de servicios de salud que permitan de manera controlada el flujo de pacientes a través de los diferentes niveles de atención.

\section{El papel del Médico Familiar como "puerta de entrada" al Sistema de Salud de cada país}

En el caso de los 16 países encuestados, sólo nueve refirieron que el paso obligado para el acceso a los médicos especialistas y demás servicios hospitalarios, es a través del Médico Familiar y/o General. En los casos de Argentina, Colombia, El Salvador, Nicaragua, Perú, Paraguay y Venezuela, se indica que los pacientes pueden acceder a los servicios médicos especializados sin que necesariamente participe el médico de familia o general (Tabla 2).

Tabla 2. El Médico Familiar como puerta de entrada al sistema de salud

Obligado el paso por el 1ํNivel de Atención

Pueden acceder directamente a especialidades
Bolivia, Brasil, Chile, Costa Rica, Cuba, México, Panamá, Puerto Rico y República Dominicana

Argentina, Colombia, El Salvador, Nicaragua, Perú, Paraguay y Venezuela

\section{La regionalización y adscripción por consultorio de Medicina Familiar o General}

Al respecto de la regionalización y adscripción de la población para cada médico familiar o general, encontramos que 11 de los 16 países encuestados (Bolivia, Brasil, Chile, Costa Rica, Cuba, El Salvador, México, Paraguay, Puerto Rico, República Dominicana y Venezuela), refirieron tener adscrita su población por consultorio, de acuerdo a una distribución geográfica, variando desde los 1500 pacientes en Cuba; 2500 en México y República Dominicana y en el caso de Bolivia es superior a los 10,000 en las diferentes instituciones de salud. En los casos de Argentina, Colombia, Nicaragua, Panamá y Perú, no ha sido posible llevar a cabo dicha regionalización, o se ha hecho de forma parcial.

\section{El SRCRP a nivel interinstitucional}

En tres de los 16 países no se pueden llevar a cabo las referencias de pacientes interinstitucionales (Bolivia, El Salvador y República Dominicana). En los 13 países restantes, se puede realizar la referencia interinstitucional bajo diferentes mecanismos administrativos como convenios de cooperación o pagos subrogados por los servicios prestados (Tabla 3).

En 15 de los 16 países, excepto Colombia, los procesos son muy similares, y consisten en la necesidad enviar a un paciente del primer nivel de atención a un centro hospitalario, ya sea para atención médica o para la realización de estudios con equipo que no existe en ese nivel de atención. Excepto Venezuela y El Salvador, todos los países manifestaron la necesidad de llenar un formulario, impreso o electrónico expresando los datos del paciente, motivo de envío y especialidad de referencia. En el caso de Paraguay, se presenta el paciente vía telefónica por el médico solicitante al de referencia hospitalaria. En el caso de México, es indispensable que los pacientes sean enviados al especialista con estudios de laboratorio y gabinete que procedan de acuerdo a los protocolos diagnósticos de cada especialidad, y en todos los casos, las referencias deben ser autorizadas (justificadas) por el jefe de la consulta. 
Tabla 3. Descripción general de los Sistemas de Referencia y Contrarreferencia de pacientes en América Latina

\begin{tabular}{|c|c|c|c|c|c|c|c|c|c|}
\hline País & $\begin{array}{l}\text { Niveles de } \\
\text { Atención } \\
\text { en AL }\end{array}$ & $\begin{array}{c}\text { Ubicación del MF } \\
\text { en los Niveles de } \\
\text { Atención }\end{array}$ & Docencia & SRCRP & $\begin{array}{l}\text { Marco } \\
\text { Jurídico }\end{array}$ & $\begin{array}{c}\text { MF como } \\
\text { Puerta de } \\
\text { Entrada al SS }\end{array}$ & $\begin{array}{c}\text { Población } \\
\text { adscrita por } \\
\text { consultorio }\end{array}$ & $\begin{array}{c}\text { SRCR } \\
\text { Interinstitucional }\end{array}$ & $\begin{array}{l}\text { Expediente } \\
\text { Clínico } \\
\text { Electrónico }\end{array}$ \\
\hline Argentina & 3 & 1 er y $2^{\circ}$ & $\mathrm{Si}$ & $\begin{array}{l}\text { No todo } \\
\text { el país }\end{array}$ & No & No & & SI & No \\
\hline Bolivia & 3 & 1 er y $2^{\circ}$ & $\mathrm{Si}$ & $\mathrm{Si}$ & $\mathrm{Si}$ & $\mathrm{Si}$ & 5000 & No & No \\
\hline Brasil & 3 & $1 \mathrm{er}$ & $\mathrm{Si}$ & $\mathrm{Si}$ & $\mathrm{Si}$ & $\mathrm{Si}$ & & $\mathrm{SI}$ & $\begin{array}{c}\text { Algunas } \\
\text { zonas }\end{array}$ \\
\hline Chile & 3 & 1 er y $2^{\circ}$ & $\mathrm{Si}$ & $\mathrm{Si}$ & $\mathrm{Si}$ & $\mathrm{Si}$ & & $\mathrm{SI}$ & $\mathrm{Si}$ \\
\hline Colombia & 3 & 1 er y $2^{\circ}$ & $\mathrm{Si}$ & $\mathrm{Si}$ & $\mathrm{Si}$ & No & & $\mathrm{SI}$ & $\begin{array}{c}\text { Algunas } \\
\text { zonas }\end{array}$ \\
\hline Costa Rica & 3 & 1er, $2^{\circ}$ y $3 e r$ & $\mathrm{Si}$ & $\mathrm{Si}$ & $\mathrm{Si}$ & $\mathrm{Si}$ & & $\mathrm{SI}$ & $\begin{array}{c}\text { Algunas } \\
\text { zonas }\end{array}$ \\
\hline Cuba & 3 & $1 \mathrm{er}$ & $\mathrm{Si}$ & $\mathrm{Si}$ & $\mathrm{Si}$ & $\mathrm{Si}$ & 1500 & SI & No \\
\hline El Salvador & 3 & 1 er y $2^{\circ}$ & $\mathrm{Si}$ & $\mathrm{Si}$ & No & No & & No & No \\
\hline México & 3 & 1 er y $2^{\circ}$ & $\mathrm{Si}$ & $\mathrm{Si}$ & $\mathrm{Si}$ & $\mathrm{Si}$ & 2500 & $\mathrm{SI}$ & $\mathrm{Si}$ \\
\hline Nicaragua & 3 & 1 er y $2^{\circ}$ & $\mathrm{Si}$ & $\mathrm{Si}$ & $\mathrm{Si}$ & No & & $\mathrm{SI}$ & No \\
\hline Panamá & 3 & $1 \mathrm{er}$ & No & $\mathrm{Si}$ & $\mathrm{Si}$ & $\mathrm{Si}$ & & $\mathrm{SI}$ & $\mathrm{Si}$ \\
\hline Paraguay & 3 & 1 er y $2^{\circ}$ & $\mathrm{Si}$ & $\mathrm{Si}$ & $\mathrm{Si}$ & No & & $\mathrm{SI}$ & No \\
\hline Perú & 3 & 1 er y $2^{\circ}$ & $\mathrm{Si}$ & $\mathrm{Si}$ & $\mathrm{Si}$ & No & & SI & No \\
\hline Puerto Rico & 3 & $1 \mathrm{er}, 2^{\circ}$ y $3 e r$ & $\mathrm{Si}$ & $\mathrm{Si}$ & $\mathrm{Si}$ & $\mathrm{Si}$ & & SI & $\mathrm{Si}$ \\
\hline $\begin{array}{l}\text { República } \\
\text { Dominicana }\end{array}$ & 3 & 1 er y $2^{\circ}$ & $\mathrm{Si}$ & $\mathrm{Si}$ & $\mathrm{Si}$ & $\mathrm{Si}$ & 2500 & No & $\begin{array}{c}\text { Algunas } \\
\text { zonas }\end{array}$ \\
\hline Venezuela & 3 & 1er & $\mathrm{Si}$ & $\mathrm{Si}$ & $\mathrm{Si}$ & No & & $\mathrm{SI}$ & No \\
\hline
\end{tabular}

\section{Los profesionales responsables de la referencia médica}

En los 16 países, el profesional responsable de la referencia es el médico generalista, sea general o especialista en familia. Resulta importante mencionar que en Bolivia, Chile, Cuba, México, Paraguay, Puerto Rico, República Dominicana y en algunas regiones de Brasil, existen profesionales responsables de validar y autorizar la solicitud de la derivación del paciente a un segundo nivel de atención.

\section{Mecanismos de control del SRCRP en las unidades de atención médica}

En sólo cuatro países de los 16 encuestados, se pudieron observar mecanismos bien definidos de control de las referencias recibidas en el hospital y de las contrarreferencias efectuadas a las unidades médicas de origen. Chile, Cuba, México y Panamá presentan mecanismos estructurados que de manera general se resumen a continuación:

Chile: toda referencia debe ser ingresada a la plataforma SIDRA lo que implica una transcripción de datos. Este sistema permite realizar una monitorización del proceso de referencia y contrarreferencia en cuanto a los tiempos de espera y asignación de horas de atención por parte del nivel $2^{\circ}$ o $3^{\circ}$. Además, se realizan reuniones de integración donde participan directivos de la APS y de la atención secundaria, en ellas se analiza la calidad de la referencia y contrarreferencia.

Cuba: existen dos instancias, una es a través del Comité de evaluación de la Calidad en APS y Hospitales y la otra consiste en reuniones de integración en la cual participan directivos de la APS y de la atención secundaria, donde se analiza la calidad de la referencia y la contrarreferencia de los pacientes.

México: existen en las diferentes instituciones de salud, áreas de control del Sistema de Referencia y Contrarreferencia que monitorean el registro de los pacientes referidos a su llegada y también a la salida cuando son contrarreferidos a sus unidades de origen, al mismo tiempo que verifican el adecuado llenado de la hoja de contrarreferencia y del otorgamiento del manejo farmacológico hospitalario para los pacientes devueltos a las clínicas de Medicina Familiar. 
Asimismo, se incluyen indicadores de calidad del Sistema de Referencia y Contrarreferencia de pacientes en las unidades de primero, segundo y tercer niveles de atención, indispensables para la certificación y recertificación de las unidades médicas.

Panamá: se confirma que la referencia realizada por medicina familiar o medicina interna, se haya recibido en el hospital a través del Departamento de Registros Médicos y Estadísticas en Salud.

\section{El manejo del expediente clínico electrónico en las unidades de primer nivel}

De los 16 países analizados, siete reportaron no contar con esa herramienta de trabajo para el primer nivel de atención (Argentina, Bolivia, Cuba, El Salvador, Nicaragua, Paraguay y Venezuela). Los otros nueve países reportaron tener diferente grado de avance en la utilización generalizada de dicho instrumento, los más consolidados parecen ser Chile, México, Panamá y Puerto Rico. Brasil lo incluye en algunas regiones del país, al igual que Perú, Colombia, República Dominicana y Costa Rica.

\section{Los sistemas electrónicos de gestión de referencia y contrarreferencia de pacientes}

Los sistemas electrónicos de referencia y contrarreferencia de pacientes para la gestión y control de citas y posteriormente la contrarreferencia, son una herramienta de los servicios de salud que actualmente se desarrollan en varios países de la región. Hasta hoy, ningún país lo tiene generalizado, de los 16 países estudiados, sólo cinco refieren su utilización de manera parcial, Chile lo hace a través del Sistema de Información de la Red Asistencial (SIDRA), Panamá a nivel de la Caja del Seguro Social (CSS) en todo el país y en el Ministerio de Salud sólo en algunos centros de salud de la capital del país. En México lo instrumenta actualmente el Instituto Mexicano del Seguro Social (IMSS). Y en el caso de Perú, sólo el Seguro Social de Salud (EsSalud).

\section{Utilización de protocolos diagnósticos o guías de práctica clínica para la referencia de pacientes}

De los 16 países, sólo 10 reportaron contar con protocolos diagnósticos o guías de práctica clínica Bolivia, Chile, Cuba, Colombia, Costa Rica, El Salvador, México, Nicaragua, Panamá y República Dominicana.

\section{Eficacia del SRCRP, entendida como porcentaje de pacientes contrarreferidos en relación al número de referidos}

En general, la mayor parte de los países no tienen los datos precisos en cuanto al porcentaje de pacientes contrarreferidos, lo que sí se puede observar es que los países que tienen mecanismos de control de las referencias y contrarreferencias en las unidades médicas, son los que refieren los mayores porcentajes de retorno de pacientes a las unidades emisoras, tal es el caso de Chile, Cuba y México.

\section{Comités de Vigilancia del SRCRP en las unidades médicas}

Encontramos que 10 de los países cuentan con Comités de Vigilancia del SRCRP, en diferentes instituciones de salud, en algunos países de manera generalizada como en el caso de Cuba, México, Puerto Rico y Perú (Seguro Social). Otros países como Bolivia, Brasil, Colombia, Costa Rica, Nicaragua y Panamá cuentan con ellos de forma irregular.

\section{Perfil de los médicos que comparten el primer nivel de atención en los países de la Región}

En países como Cuba, Chile, México y Paraguay, la mayor parte de las unidades de primer nivel de atención son atendidas por médicos generalistas, ya sean Médicos Familiares o Generales. En países como Brasil y Perú, la conformación de los equipos depende de la región geográfica. En el resto de los países también podemos encontrar la presencia de pediatras, gineco-obstetras, internistas o incluso psiquiatras, oftalmólogos o dermatólogos, etc., como es el caso de Argentina, Bolivia, El Salvador, Costa Rica, Nicaragua, Panamá, Puerto Rico, República Dominicana y 
Venezuela. En el caso de Colombia no se encuentran Médicos Familiares en el primer nivel de atención, se pueden ubicar a partir del segundo nivel colaborando igualmente con otros especialistas médicos.

\section{Los procesos de acreditación de la calidad de las unidades médicas y el SRCRP}

En la presente investigación se pudo encontrar que sólo cinco de los 16 países encuestados incluyen la medición de indicadores de referencia y contrarreferencia en los procesos de acreditación de las unidades de salud (Chile, Colombia, Cuba, México y Perú).

\section{Los programas de atención domiciliaria por el Médico Familiar y/o el equipo de Salud Familiar}

Resulta satisfactorio que no obstante las dificultades mencionadas, en 15 de los 16 países se desarrollan diferentes modalidades de atención domiciliaria, en la mayoría de los casos otorgada por el médico de familia o incluso por el médico general, y en otros por diferentes actores del equipo básico de salud familiar. En este sentido, el país que no reporta atención domiciliaria es Nicaragua.

\section{Discusión y Conclusiones}

La presente investigación nos permitió confirmar que la figura del especialista en Medicina Familiar está presente en los 16 países investigados y en los tres niveles de atención, con diversidad de funciones, principalmente encaminadas a las áreas clínica y administrativa, con menor frecuencia en actividades docentes y de investigación.

Es importante destacar que los SRCRP funcionan en los 16 países, encontrando la mayor eficiencia en la referencia de pacientes del primero al segundo y del segundo al tercer nivel de atención. Sin embargo, las mayores dificultades se observan en la contrarreferencia, situación que limita la retroalimentación de los médicos de atención primaria, e incluso, a los del segundo nivel.

Resulta preocupante que algunos países cuentan con modelos de salud incongruentes, que no corresponden al discurso político y a las propias reformas que promueven a través de sus respectivos gobiernos, incluso, en el tipo y cantidad de profesionales de salud especializados en Medicina Familiar y Atención Primaria, tal como se recomienda por los organismos internacionales a nivel mundial. ${ }^{1,5,6}$

Como se mencionó al inicio, los procesos de evaluación de la calidad de las unidades de atención médica, incluyen dentro de sus indicadores los relacionados con la eficacia y eficiencia del SRCRP. No obstante, en los últimos años se ha impulsado desde los foros mundiales de salud la Certificación o Acreditación de la Calidad de los establecimientos de salud, que permitan mejorar la práctica médica. ${ }^{1,2}$ Los países de América Latina han avanzado en su instrumentación paulatina, incorporando a sus sistemas de evaluación de la calidad de la práctica médica, la formación de Comités de vigilancia; la creación de protocolos de diagnóstico y tratamiento; reuniones de retroalimentación entre los diferentes niveles de atención, acerca del tipo y calidad de las referencias; etcétera. Los países que mejor eficacia y eficiencia en sus sistemas de referencia y contrarreferencia tienen, son los que han alcanzado mayor consolidación en sus procesos de acreditación de la calidad de sus servicios de salud, incorporando en él, indicadores del SRCRP.

Finalmente, el grupo de trabajo recomienda las siguientes estrategias de fortalecimiento para los SRCRP en la Región:

- Incrementar y mejorar la calidad de los programas de formación de especialistas en Medicina Familiar.

- Garantizar la presencia de especialistas en Medicina Familiar laborando en el primer nivel de atención, en el área clínica, de gestión, de docencia y de investigación, lo que permitirá incrementar la capacidad resolutiva de las unidades médicas.

- Establecer Sistemas de Certificación y Recertificación de la Calidad de unidades médicas en los tres niveles de atención, que incorporen indicadores de eficacia y eficiencia del SRCRP.

- Implantar sistemas de expediente electrónico único, que permita su portabilidad en las diferentes instituciones de salud y que esté vinculado al SRCRP en los tres niveles de atención. 


\section{Referencias}

1. Organización Mundial de la Salud, Informe sobre la Salud en el Mundo. La financiación de los Sistemas de Salud, El camino hacia la cobertura universal. Organización Mundial de la Salud (OMS). Ginebra. 2010. Consultado el 17-de marzo de 2016. Disponible en: http://apps.who.int/iris/bitstream/10665/44373/1/9789243564029_spa.pdf

2. World Health Organization. Everybody's Business: Strengthening Health Systems to improve health Outcomes. WHO Framework for Action. Geneva, 2007

3. Frenk, J. Las dimensiones de la Reforma del Sistema de Salud. En Ruiz Durán, Clemente. Los sistemas de seguridad social en el siglo XXI. México D.F. 1997

4. Gómez A, Pineda F. Sistemas de salud: Definiciones, componentes y evaluación. Monitor Estratégico, Superintendencia Nacional de Salud de Colombia. No. 4 Jul-Dic. 2013. p 85-92

5. Fortalecimiento de los sistemas de salud, Tendencias actuales y retos, 64. A Asamblea Mundial de la Salud, OMS. Documento A64/13. Ginebra: OMS, 2011. Consultado el 17 de marzo de 2016. Disponible en: http://apps.who.int/gb/ebwha/pdf_files/ WHA64/A64_13-sp.pdf

6. Organización Panamericana de la Salud/Organización Mundial de la Salud. La Renovación de la Atención Primaria de la Salud en las Américas. Documento de posición de la Organización Panamericana de la Salud, Organización Mundial de la Salud (OPS/OMS). Washington, DC, 2007.

7. Sistema Integral de Referencia y Contrarreferencia. Secretaría de Salud. Alcaldía Mayor de Bogotá. Colombia, 2007. Consultado el Disponible en: http://www.alcaldiabogota.gov.co/sisjur/normas/Norma1.jsp?i=27905

8. Manual Operativo del Sistema de Referencia y Contrarreferencia. Secretaría Departamental de Salud del Valle del Cauca. Colombia, 2014. Consultado el 17 de marzo de 2016. Disponible en: file:///C:/Users/Miguel/Downloads/MANUAL_ SIRC_2014\%20(1).pdf

9. Soberón G. La Reforma de Salud en México. Gac Méd Méx. Vol. 137 No. 5, 2001; 419-4

10. Stoner J, Freeman E, Gilbert Jr D. Administración. 6ạ ed. Prentice Hall. 1996; 35-42.

11. Propuesta de Directiva de Sistema de Referencia y Contrarreferencia en la Atención Integral de la Tuberculosis y Enfermedades respiratorias (PAL). Ministerio de Salud de Perú, 2012. Consultado el 17 de marzo de 2016. Disponible en: http://www. parsalud.gob.pe/phocadownload/fondomundial/ProdcutoMaterialIEC/PAL/Directiva\%20Sistema\%20de\%20Referncia\%20 y\%20Contrareferencia\%20version\%20final\%20validada.pdf

12. Lineamientos para la Referencia y Contrarreferencia de pacientes pediátricos de población abierta de la Ciudad de México y área conurbada del Estado de México. Secretaría de Salud del Distrito Federal. 2002. Consultado el 17 de marzo de 2016. Disponible en: http://www.salud.gob.mx/unidades/cdi/documentos/DOCSAL7455.pdf

13. Norma Técnica del Sistema de Referencia y Contrarreferencia de los establecimientos del Ministerio de Salud. Ministerio de Salud de Perú. 2005. Consultado el 17 de marzo de 2016. Disponible en: http://bvs.minsa.gob.pe/local/dgsp/115_NTREFYCON.pdf

14. Barquín M. Dirección de hospitales. 6ạ ed. Interamericana Mc Graw Hill. 1992; 25-35.

15. Barquín M. Niveles de complejidad de la atención médica. En: Sociomedicina. 4ạ ed. Méndez Editores. 1994; 615-628.

16. Caballero A, et al. Comunicación entre atención primaria y atención especializada: análisis de los contenidos de derivación y retorno de enfermos reumatológicos. Atención Primaria. Vol.12. Núm.5. 30 de septiembre de 1993; 30-35.

17. Silverman M. Optimización del proceso de Referencia y Contrarreferencia entre los niveles de la atención. Evaluación de la implementación de un sistema de comunicación electrónico. Maestría en Salud Pública, orientación en Sistemas Municipales. Universidad Nacional de la Plata. Argentina. 2007. Consultado el 17 de marzo de 2016. Disponible en: http://www.postgradofcm. edu.ar/ProduccionCientifica/TesisMagisters/7.pdf

18. Manual de Organización de las Unidades Médicas del Primer Nivel de Atención. ISSSTE. Octubre de 2000; 9-13.

19. Levy S. La modernización del Instituto Mexicano del Seguro Social. Gac Méd Méx. Vol. 137 No.5, 2001; 425-428.

20. Pablo M, Abreu M, Antonietti L, Guillén M, Said M, Montali C. Sistemas de referencia y contrarreferencia entre el segundo y el tercer nivel de salud en una red hospitalaria pediátrica en el conurbano bonaerense. Arch Argent Pediatr 2013; 111 (5):405-411 Consultada 17 de marzo de 2016. Disponible en: http://www.postgradofcm.edu.ar/ProduccionCientifica/TesisMagisters/7.pdf 